\section{Does the computed tomography appearance of the lungs of old dogs differ from those of young dogs?}

\section{Natasha Hornby, Chris Lamb}

Royal Veterinary College, London, UK

\section{OBJECTIVES}

To determine if there are differences in the computed tomography (CT) appearance of the lungs of young and old dogs that could affect interpretation of diagnostic studies.

\section{METHODS}

Retrospective review of pulmonary CT images of dogs with benign conditions unrelated to the thorax. CT images of young (<5years) and old (>11years) dogs were collected, ordered randomly and reviewed with respect to twelve different variables by a Board certified radiologist blinded to age. Mann-Whitney, Chi-square and Kendall's tau-c statistics were used to test differences.

\section{RESULTS}

CT images of 42 young dogs (range $0.3-4.8$ years) and 47 old dogs (range 11-15.1 years) were reviewed. CT was performed under sedation in 62 (70\%) dogs and under general anaesthesia in 27 (30\%). Heterotopic bone was more prevalent $(47 \%$ versus $7 \% ; \mathrm{p}<0.001)$ and lung collapse was observed more frequently $(58 \%$ versus $31 \%$; $p=0.003)$ in old dogs. There were no significant differences in lung attenuation, reticular pattern, ground glass pattern, emphysema, pleural thickening, septal thickening, bronchial thickening, bronchial dilation, tracheal calcification or presence of cysts.

\section{STATEMENT}

Despite previous studies describing age-related changes in the radiographic appearance of the lungs of old dogs, it appears that there are minimal observable differences in CT images. Old dogs are more likely to have heterotopic bone and may be more prone to lung lobe collapse than young dogs, but neither of these differences should contribute to misdiagnosis of pulmonary disease. 